\title{
Multi-response optimization of Wire-EDM process using principal component analysis
}

\author{
Miss.Swati.D.Lahane ${ }^{1}$, Prof.Manik.K.Rodge ${ }^{2}$ Dr. Sunil.B. Sharma ${ }^{3}$ \\ ${ }^{I}$ (S.G.G.S.I.E\&T, Dept. of production engg.Nanded.) \\ ${ }^{2}$ (S.G.G.S.I.E\&T, Dept. of production engg. Nanded.) \\ ${ }^{3}$ (S.G.G.S.I.E\&T, Dept. of production engg.Nanded.)
}

\begin{abstract}
Wire electrical discharge machining (WEDM) has been found to be an extremely potential electro-thermal process in the field of conductive material machining. This process employed for the parts demanding higher accuracy levels with varying hardness or complex shapes, which have sharp edges that are very difficult to be machined by main stream processes.

WEDM is a complex machining process controlled by a large number of process parameters such as the pulse duration, discharge frequency and discharge current density. Any slight variations in the process parameters can affect the machining performance measures such as surface roughness and cutting ratio.

Taguchi method is widely used for optimization of various processes. Using Taguchi method, the parametric settings can be optimized with respect to one performance characteristic (response) at a time, whereas wire electrical discharge machining (WEDM) processes require optimization of multiple performance characteristics. Researchers have attempted several approaches but determination of the optimal process settings that can optimize multiple performance measures (responses) of WEDM operations still remains an important issue. In this paper, weighted principal component (WPC) method is used to optimize the multiple responses of WEDM processes. The results show that the WPC method offers significantly better overall quality than the other approaches.
\end{abstract}

Keywords: - Wire electrical discharge machining (WEDM), weighted principal component (WPC),

\section{INTRODUCTION}

Wire electrical discharge machining (WEDM) has been found to be an extremely potential electro-thermal process in the field of conductive material machining. This process employed for the parts demanding higher accuracy levels with varying hardness or complex shapes, which have sharp edges that are very difficult to be machined by main stream processes.

In this operation, the material removal occurs from an electrically conductive material by initiation of rapid and repetitive spark discharges between the gap of the work and tool electrode connected in an electrical circuit .

There is no relative contact between the tool and work, the work material hardness is not a limiting factor for machining materials by this process. Because of its higher process capability and productivity WEDM is an important machining process to produce complex and intricate shapes of components in areas such as tool and die making, automobile, aerospace, nuclear, computer and electronics industries, etc. with the highest degree of dimensional accuracy and surface finish quality.

The material removal mechanisms of EDM and WEDM are similar, but their functional characteristics are not identical. WEDM uses a thin wire continuously feeding through the workpiece by a microprocessor control which enables parts of complex shapes to be machined with exceptionally higher accuracy. A varying degree of taper ranging from $15^{\circ}$ for a $100 \mathrm{~mm}$ thick to $30^{\circ}$ for a $400 \mathrm{~mm}$ thick workpiece can also be obtained on cut surface. The microprocessor constantly maintains gap between the wire and the workpiece $(0.025$ to 0.05 $\mathrm{mm})$.

\subsection{Maxi cut WEDM}

\section{Machine Specification}

\section{CONCEPT OF 4-AXIS WIRE CUT EDM}

The ELEKTRA WIRE CUT Electric Discharge Machine comprises of a Machine Tool, a Power Supply Unit (ELPULS) and a Dielectric Unit.

The machine tool comprises of a main worktable (called X-Y table) on which the workpiece is clamped, an auxiliary table (called $\mathrm{U}-\mathrm{V}$ table) and wire drive mechanism. The main table moves along $\mathrm{X}$ and $\mathrm{Y}$ axes, in steps of 1 micrometer, by means of $\mathrm{X}$ and $\mathrm{Y}$ pulse motors, whereas the U-V table moves, in steps of 1 
micrometer, by means of $\mathrm{U}$ and $\mathrm{V}$ pulse motors along $\mathrm{U}$ and $\mathrm{V}$ axes which are parallel to $\mathrm{X}$ and $\mathrm{Y}$ axes respectively.

The Power Supply Unit (ELPULS) comprises of -

1. $\mathrm{U}-\mathrm{V}$ controller with $\mathrm{U}-\mathrm{V}$ axes drive and position display.

2. Main X-Y controller with $\mathrm{X}$ and $\mathrm{Y}$ position and machining block number display.

3. Machining pulse parameter and servo control unit.

4. X-Y motor driver unit.

5. Machining pulse generator unit

A series of electrical pulses generated by this pulse generator unit are applied between the workpiece and the traveling wire electrode, to cause the electro-erosion of the workpiece material. As the material removal or machining proceeds, the worktable carrying the workpiece is displaced transversely by the $\mathrm{X}-\mathrm{Y}$ controller and driver along a predetermined path programmed in the controller.

The path specifications can be supplied to the controller via a preprogrammed buffer or directly through the controller keyboard (MDI mode). When the $\mathrm{X}-\mathrm{Y}$ table is moving along the predetermined path, if the U-V table is kept stationary, a straight cut with a predetermined pattern is formed. [9]

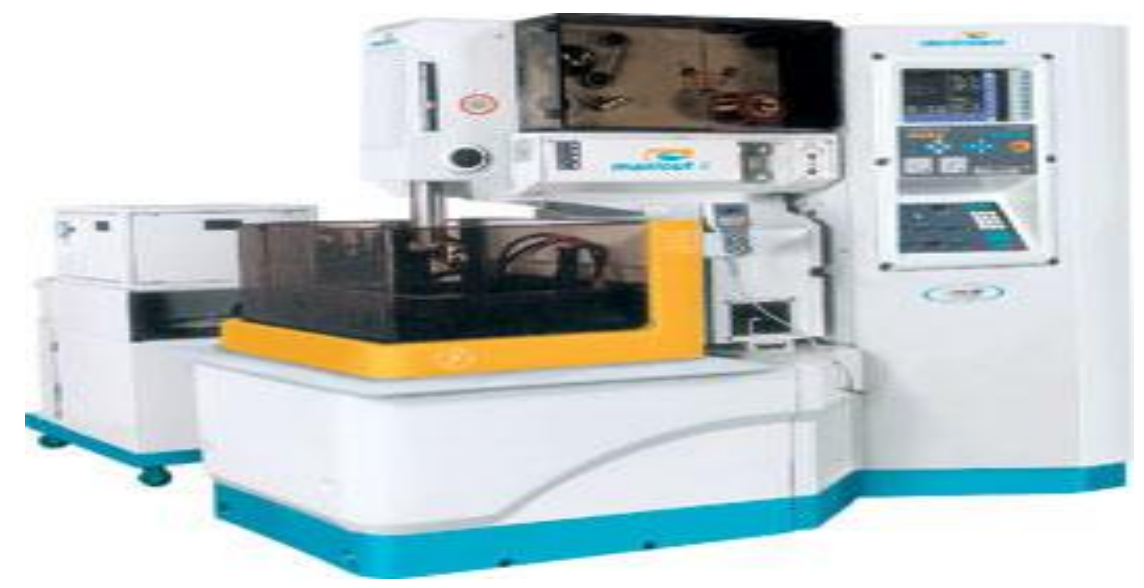

\section{TECHNICAL SPECIFICATIONS}

\section{Machine Tool}

Max. table size

Max.workpiece height

Max. workpiece weight

Main table traverse $(\mathrm{X}, \mathrm{Y})$

Auxiliary table traverse (u, v)

Max. taper cutting angle

Max. wire spool capacity

Dry run speed

Resolution

Wire diameter
$440 \times 650 \mathrm{~mm}$

$200 \mathrm{~mm}$

$300 \mathrm{~kg}$

$300,400 \mathrm{~mm}$

$80,80 \mathrm{~mm}$

$\pm 15 \% 100 \mathrm{~mm}$

$6 \mathrm{~kg}$

$80 \mathrm{~mm} / \mathrm{min}$

$0.001 \mathrm{~mm}$

$0.25 \mathrm{~m}$ (Standard)

0.15, 0.2, $0.3 \mathrm{~mm}$ (Optional) 
Multi-response optimization of Wire-EDM process Using principal component analysis

\section{Literature Review}

WEDM is a complex machining process controlled by a large number of process parameters such as the pulse duration, discharge frequency and discharge current density. Any slight variations in the process parameters can affect the machining performance measures such as surface roughness and cutting ratio.

Y. Chen and S. M. Mahdivian developed a theoretical model to estimate the MRR and surface quality of workpiece. Experiments with different values of discharge current, pulse duration time and interval time were conducted to investigate their effects on the surface finish of the workpiece and material removal rates. The theoretical prediction and experimental results are in agreement when compared.

Among the different material removal processes, WEDM is considered as an effective and economical tool in machining of modern composite materials. Several comparative studies have been made between WEDM and laser cutting in the possessing of metal matrix composites (MMC), carbon fiber and reinforced liquid crystal polymer composites. These studies showed that, WEDM yields better cutting edge quality and has better control of the process parameters with fewer workpiece surface damages. However, it has lower MRR for all the tested composite materials. Other studies show that WEDM of $\mathrm{Al}_{2} \mathrm{O}_{3}$ particulate reinforced composites investigating the effect of the process parameters on the WEDM performance measures was found that the process parameters have little influence on the surface roughness but have an adverse effect on CR. As compared to conventional diamond sawing, WEDM produces a roughness and hardness that is comparable to a low speed diamond saw but with a higher MRR.

The presence of the hard reinforcing ceramics in the metal matrix composites makes them difficult for conventional machining. A Gatto and L. Iuliano [5] performed WEDM tests under one roughing and two finishing conditions on two composites, SiC/2009Al alloy with $15 \%$ whiskers and with $20 \%$ particles reinforcement. Some roughed and some finished surfaces were glass-bead peened. The experiments show that WEDMing rates $(\mathrm{mm} / \mathrm{min})$ of both composites are equal. The roughness values of the WED-machined surfaces of the $15 \% \mathrm{SiCw} / 2009 \mathrm{Al}$ alloy composite are less than the corresponding values for the surfaces of the $20 \% \mathrm{SiCp} / 2009 \mathrm{Al}$ alloy composite. $\mathrm{SiC}$ reinforcement and $\mathrm{Cu}$ precipitates are absent in the outer re-cast layer. Under finishing conditions and for the both of the composites, the thickness of the outer layer without reinforcement is less than $5 \mu \mathrm{m}$. The glass-bead peening caused the disappearance of the layer without reinforcement.

Susanta Kumar Gauri \& Shankar Chakraborty[4] researched Like many other processes, the wire electrical discharge machining (WEDM) process has several performance characteristics. Determination of the optimal process settings with respect to all these performance measures (responses) is an important issue. In this paper, some modifications in the PCA based approach are suggested and two sets of experimental data published by the past researchers are analysed using this modified procedure. It is observed that the PCA-based optimisation can give better results than the constrained optimisation and MRSN ratio-based methods, which can be attributed to the fact that the possible correlation among the Multiple responses are taken care in the PCA-based approach.

Taguchi method is widely used for optimisation of various processes. Using Taguchi method, the parametric settings can be optimised with respect to one performance characteristic (response) at a time, whereas wire electrical discharge machining (WEDM) processes require optimisation of multiple performance characteristics. Researchers have attempted several approaches but determination of the optimal process settings that can optimise multiple performance measures (responses) of WEDM operations still remains an important issue. In this paper, weighted principal component (WPC) method is used to optimize the multiple responses of WEDM processes. The results show that the WPC method offers significantly better overall quality than the other approaches.

\section{Objective Experimental Study}

The experiments were performed on Maxicut-e highprecision 5 axis CNC WEDM, which is manufactured by Electronica Pvt Ltd, pune. The basic parts of the WEDM machine consists of a wire, a worktable, a servo control system, a power supply and dielectric supply system. The maxicut-e allows the operator to choose input parameters according to the material and height of the workpiece and tool material from a manual provided by the WEDM manufacturer. The maxicut-e WED machine has several special features. The pulse power supply uses a transistor controlled RC circuit. The discharge energy is determined by the value of the capacitor that is parallel to the machining gap. The experimental set-up for the data acquisition of the sparking frequency and machine table

Speed is illustrated in machine specification. 


\subsection{Material, test conditions and measurement High Speed Steel (HSS)}

- $\quad$ 1. HSS are obtained by alloying tungsten, chromium, vanadium, cobalt \& molybdenum with steel etc.

- 2. HSS is high temp. Resistance, hard \& wear resistance tool material.

Workpiece Chemical Composiontion (Wt. \%)

$\begin{array}{ll}\text { ELEMENT } & \text { WEIGHT\% } \\ \text { Carbon } & 0.874 \\ \text { Silicon } & 0.338 \\ \text { Mn } & 0.172 \\ \text { Sulphur } & 0.021 \\ \text { P } & 0.023 \\ \text { Cromium } & 3.815 \\ \text { Nickel } & 0.157 \\ \text { Molebdenum } & 4.526 \\ \text { Cupper } & 0.185 \\ \text { Ferrus } & 80.064 \\ \text { Titanium } & 0.004 \\ \text { Alluminium } & 0.025 \\ \text { V } & 1.752 \\ \text { Cupper } & 1.014 \\ \text { W } & 5.568 \\ \text { Pb } & 0.022 \\ \text { Nb } & 0.026\end{array}$

To optimize the process parameters of WEDM such as

\begin{tabular}{|l|l|l|l|l|l|l|}
\hline Sr.No. & Parameters & Unit & Symbol & \multicolumn{2}{|l|}{ Values of Levels } \\
\hline & & & & 1 & 2 & 3 \\
\hline \hline $\mathbf{1}$ & Pulse on time(Ton) & micro-sec & A & $\mathbf{3}$ & $\mathbf{5}$ & $\mathbf{7}$ \\
\hline $\mathbf{2}$ & Pulse off time(Toff) & micro-sec & B & $\mathbf{4}$ & $\mathbf{6}$ & $\mathbf{8}$ \\
\hline $\mathbf{3}$ & Upper Flush & $\mathbf{K g} / \mathbf{c m}^{\wedge} \mathbf{2}$ & $\mathbf{C}$ & $\mathbf{1 0}$ & $\mathbf{8}$ & $\mathbf{6}$ \\
\hline $\mathbf{4}$ & Wire feed & $\mathbf{m m} / \mathbf{m i n}$ & $\mathbf{D}$ & $\mathbf{5}$ & $\mathbf{7}$ & $\mathbf{9}$ \\
\hline & & & & & & \\
\hline
\end{tabular}

For more MRR \& less WWR of the HSS material .

\section{Key objective:}

- Finding out Optimum Value for above parameters.

\subsection{Typical Tools In WEDM:}

- Copper Wire used as electrode of $0.25 \mathrm{~mm}$ diameter

- HSS material used as work piece

- Dielectric water is used as cooling material 


\section{Design of Experiments}

A well planned set of experiments, in which all parameters of interest are varied over a specified range, is a much better approach to obtain systematic data. Mathematically speaking, such a complete set of experiments ought to give desired results.

The analysis is not very easy (though it may be easy for the mathematician/statistician) and thus effects of various parameters on the observed data are not readily apparent. In many cases, particularly those in which some optimization is required, the method does not point to the BEST settings of parameters. A classic example illustrating the drawback of design of experiments is found in the planning of a world cup event, say football. While all matches are well arranged with respect to the different teams and different venues on different dates and yet the planning does not care about the result of any match (win or lose)!!!! Obviously, such a strategy is not desirable for conducting scientific experiments (except for co-ordinating various institutions, committees, people, equipment, materials etc.).

\subsection{Taguchi Method}

Dr. Taguchi of Nippon Telephones and Telegraph Company, Japan has developed a method based on "ORTHOGONAL ARRAY" experiments which gives much reduced " variance " for the experiment with " optimum settings " of control parameters.

Thus the marriage of Design of Experiments with optimization of control parameters to obtain BEST results is achieved in the Taguchi Method. "Orthogonal Arrays" (OA) provide a set of well balanced (minimum) experiments and Dr. Taguchi's Signal-to-Noise ratios $(\mathrm{S} / \mathrm{N})$, which are log functions of desired output, serve as objective functions for optimization, help in data analysis and prediction of optimum results

\subsection{Introduction to Taguchi Method}

Every experimenter has to plan and conduct experiments to obtain enough and relevant data so that he can infer the science behind the observed phenomenon. He can do so by,

Trial-and-error approach performing a series of experiments each of which gives some understanding. This requires making measurements after every experiment so that analysis of observed data will allow him to decide what to do next - "Which parameters should be varied and by how much".

Many a times such series does not progress much as negative results may discourage or will not allow a selection of parameters which ought to be changed in the next experiment. Therefore, such experimentation usually ends well before the number of experiments reaches a double digit! The data is insufficient to draw any significant conclusions and the main problem (of understanding the science) still remains unsolved.

\subsection{Taguchi views}

Engineering design process having three phases

1) System design 2) Parameter design 3) Tolerance design

Two major tools used in Taguchi

1) Orthogonal arrays which are used to study many parameters.

2) Signal to noise ratio which measures quality.

This method is an engineering approach of quality improvement that seeks to obtain a lowest cost solution to the product design specification based on the requirements of the customer.

\subsection{DOE-according to Taguchi method}

\section{L-9 Array}

\begin{tabular}{|l|l|l|l|l|}
\hline Sr.No & T-on & T-off & UF & Wirefeed \\
\hline 1 & 3 & 4 & 10 & 5 \\
\hline 2 & 3 & 6 & 8 & 7 \\
\hline 3 & 3 & 8 & 6 & 9 \\
\hline 4 & 5 & 4 & 8 & 9 \\
\hline 5 & 5 & 6 & 6 & 5 \\
\hline 6 & 5 & 8 & 10 & 7 \\
\hline 7 & 7 & 4 & 6 & 7 \\
\hline 8 & 7 & 6 & 10 & 9 \\
\hline 9 & 7 & 8 & 8 & 5 \\
\hline
\end{tabular}




\section{Experimentation}

\begin{tabular}{|l|l|l|l|l|}
\hline $\begin{array}{l}\text { Sr.n } \\
\mathbf{o}\end{array}$ & $\begin{array}{l}\text { Voltage(v } \\
\text { ) }\end{array}$ & $\begin{array}{l}\text { Current } \\
(\mathbf{a m p})\end{array}$ & $\begin{array}{l}\mathbf{m} / \mathbf{c} \text { speed } \\
(\mathbf{m m} / \mathbf{m i n})\end{array}$ & remarks \\
\hline 1 & $70-72$ & 1.5 & $0.6-0.5$ & Continuous cutting \\
\hline 2 & $66-72$ & 1.5 & $0.4-0.5$ & Continuous cutting \\
\hline 3 & $74-76$ & 1.3 & $0.3-0.4$ & Continuous cutting \\
\hline 4 & $68-72$ & 1.6 & $0.4-0.5$ & Continuous cutting \\
\hline 5 & $60-74$ & 1.6 & $\begin{array}{l}0.4-0.6 \\
(\text { vibration })\end{array}$ & Continuous cutting \\
\hline 6 & $68-72$ & 1.4 & $0.3-0.4$ & Continuous cutting \\
\hline 7 & $72-74$ & $1.6-1.7$ & $\begin{array}{l}0.5-0.6 \\
(\text { more } \\
\text { fluctuation })\end{array}$ & Continuous cutting \\
\hline 8 & $72-74$ & 1.6 & $0.5-0.6$ & Continuous cutting \\
\hline 9 & 70 & 1.0 & 0.5 & Continuous cutting \\
\hline
\end{tabular}

\section{Result of experiment (MRR, WWR)}

7.1 MRR (material removal rate)-

$\mathbf{M R R}=\mathbf{C s} * \mathbf{L} \quad$ where- $\mathrm{Cs}=$ cutting speed in $\mathrm{mm} / \mathrm{min}$

$\mathrm{L}=$ thickness of $\mathrm{w} / \mathrm{p}$ unit $(\mathrm{mm} 2 / \mathrm{min})$

7.2 WWR (wire wear rate)-

WWR = WWL / IWW Where- WWL=loss of wire wt. IWW= initial wire wt.

\begin{tabular}{|l|l|l|}
\hline Sr.no. & MRR (mm2/min) & WWR \\
\hline 1 & 1.0 & 0.95 \\
\hline 2 & 0.9 & 0.97 \\
\hline 3 & 0.7 & 0.95 \\
\hline 4 & 1.0 & 0.95 \\
\hline 5 & 1.0 & 0.95 \\
\hline 6 & 0.8 & 0.90 \\
\hline 7 & 1.2 & 0.97 \\
\hline 8 & 1.2 & 0.95 \\
\hline 9 & 1.0 & 0.97 \\
\hline
\end{tabular}

VIII. Weighted principal component (WPC) method

8.1Why need of wpc

- Taguchi's robust design method only applied to optimize a single-response problem.

- Research shows that the multi-response problem is still an issue with the taguchi method. 
- $\quad$ some researchers have attempted to optimize WEDM operations using MRSN ratio \& constraint optimization methods.

Principal component analysis (PCA) is used to explain the variance-covariance structure of a set of

variables by linearly combining the original variables. The PCA technique can account for most of the variation of the original $\mathrm{p}$ variables via $\mathrm{q}$ uncorrelated principal components, where $\mathrm{q} \leq \mathrm{p}$. In the context of Taguchi's robust design experimentation, let there be $\mathrm{m}$ experimental trials, and in each trial, quality losses of a set of $\mathrm{p}$ performance characteristics (response variables) are measured. Therefore, (L) $\mathrm{m} \times \mathrm{p}$ will be the experimental data set. Taguchi categorized the performance characteristics (response variables) into three different types, e.g. the smaller-the-better, the larger-the-better and nominal the best. The formulae for computation of quality loss (Lij) for jth response corresponding to ith trial $(\mathrm{i}=1,2 \ldots \mathrm{m} ; \mathrm{j}=1,2 \ldots \mathrm{p})$ are different for different types of response variables, and these are given as follows:

For smaller-the-better,

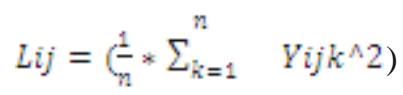

For larger-the-better,

$$
L i j=\left(\begin{array}{l}
1 \\
-
\end{array} \sum_{k=1}^{n n}\left(1 / Y_{i j} k^{n} 2\right)\right)
$$

For nominal-the-best,

$$
\begin{gathered}
L_{i j}=\left(\frac{\mu^{2}}{\sigma^{2}}\right) \\
\text { where, } \mu=\frac{1}{n} \sum_{k=1}^{n} y_{i j k}, \sigma^{2}=\frac{1}{n-1} \sum_{k=1}^{n}\left(y_{i j k}-\mu\right)^{2},
\end{gathered}
$$

, $\mathrm{n}$ represent the number of repeated experiments, yijk is the experimental value of jth response variable in ith trial at kth test and $\mathrm{Lij}$ is the computed quality loss for jth response in ith trial.

Through the PCA, the variability in the original $\mathrm{p}$ response variables corresponding to ith trial can be explained by the following q uncorrelated linear combinations:

$$
\Omega_{l}^{i}=a_{l 1} L_{i 1}+a_{l 2} L_{i 2}+\ldots+a_{l p} L_{i p} ;(l=1,2, \ldots, q)
$$

Further, Wi1 is called the first principal component, Wi2 is the second principal component and so on.

The coefficients of the lth component, i.e. al1, al2, alp are the elements of the eigenvector corresponding to the lth eigenvalue of the correlation matrix or the covariance matrix of the response variables. The eigenvalues for the $\mathrm{q}$ components and the eigenvector corresponding to each eigenvalue can be obtained by subjecting the experimental data set, (L)m $\times p$, to principal component analysis. The option of performing the PCA is available in SAS, SPSSX and STATISTICA software.

The eigenvalue of a principal component gives a fairly good idea about the variance of the original variables that can be explained by the principal component. A larger eigenvalue of a principal component implies that the component's contribution in explaining the overall variation is higher. According to Su and Tong and Antony, the components with eigenvalues greater than 1 may be selected to replace the original responses. They further observed in their case studies that the eigenvalue for the first principal component is only bigger than 1 and therefore they took into consideration the first principal component only. It may be noted that the computed first principal component, W1, corresponding to a trial is essentially the weighted average of the original $\mathrm{p}$ response

variables, where the weights are analytically decided instead of the engineer's judgment. Regarding the first principal component as an index (multi-response performance statistic), they optimised the process settings. However, problems can arise in the situations where more than one eigenvalue becomes greater than 1 . For such situations, according to Su and Tong, trade-off is necessary to select a feasible solution. However, the required trade-off for a feasible solution is unknown.

The weighted principal component (WPC)-based procedure for optimisation of multi-response processes makes use of all the principal components irrespective of the eigenvalues so that the overall variation in all the responses can be completely explained. In this approach, the proportion of overall variation explained by each component is treated as the weight to combine all the principal components in order to form a multiresponse performance index (MPI). Then the best combination of the parametric settings can easily be obtained that can optimize the MPI. It may be noted that Liao applied the WPC method 
Considering computed quality losses, i.e. (L)m×p as the experimental data. Here it is suggested to compute the signal-to-noise $(\mathrm{S} / \mathrm{N})$ ratio, which is the logarithmic transformation of the quality-loss function, for each response in each trial. This is because the logarithmic transformation improves additivity of effects of two or more control factors . On the other hand, if the actual value of some performance characteristic, e.g. defect count is used for constructing the additive model, it is quite possible that the predicted value of the defect count under the optimal conditions will become negative. This is highly undesirable since negative counts are meaningless. In the logarithmic scale, such negative counts can never occur. The $\mathrm{S} / \mathrm{N}$ ratio is always expressed in decibel (dB) unit. Since log is a monotone function, minimization of quality loss is equivalent to maximization of S/N ratio.

The WPC method for multi-response optimization can, thus, be described in the following five steps:

\section{Step 1: Compute the signal-to-noise $(\mathrm{S} / \mathrm{N})$ ratio for each response.}

Depending upon the type of the quality characteristic, calculate the quality loss (Lij) of jth response corresponding to ith trial using Eq. (1) or (2) or (3), and then, compute the $\mathrm{S} / \mathrm{N}$ ratio ( $\eta \mathrm{ij}$ ) value for jth response corresponding to ith trial using Eq. (5) as shown below:

$$
\eta_{i j}=-10 \log _{10} L_{i j}
$$

Step 2: Transform the $\mathrm{S} / \mathrm{N}$ ratio values for each response into $(0,1)$ interval.

The scaling of $\mathrm{S} / \mathrm{N}$ ratio values for each response reduces the variability among those values for different responses. $\eta \mathrm{ij}$ is transformed into $\mathrm{Yij}(0 \leq \mathrm{Yij} \leq 1)$ using the following equation:

$$
Y_{i j}=\frac{\eta_{i j}-\eta_{j}^{\min }}{\eta_{j}^{\max }-\eta_{j}^{\min }}
$$

Where, $\mathrm{Yij}=$ scaled $\mathrm{S} / \mathrm{N}$ ratio value for jth response at ith trial,

Step 3: Perform the PCA on the computed data, (Yij) m×p and obtain the principal components.

As mentioned earlier, the option of performing the PCA is available in SAS, SPSSX and STATISTICA software. Subject the data set, (Yij)m $\times p$, to principal component analysis and obtain the eigenvalues of the $q$ principal components and the eigenvector corresponding to each eigenvalue. Then, similar to Eq. (4), obtain the q principal components corresponding to a trial (i) as follows:

$$
Z_{l}^{i}=a_{l 1} Y_{i 1}+a_{l 2} Y_{i 2}+\ldots+a_{l p} Y_{i p} ;(l=1,2, \ldots, q)
$$

Step 4: Compute the multi-response performance index (MPI) corresponding to each trial.

The multi-response performance index (MPI) is essentially the weighted sum of all the principal components. The MPI value for ith trial, therefore, can be computed using the following equation:

$$
\mathrm{MPI}^{\mathrm{i}}=\sum_{l=1}^{q} W_{l} Z_{l}^{i}
$$

Where, $\mathrm{Wl}$ is the proportion of overall variance of the responses explained by lth principal component, $\mathrm{Zi} 1$ is the computed value of lth principal component correspondingto ith trial and It may be noted that since all the principal components are independent of each other, the additive model is appropriate here.

"A larger value of MPI will imply better quality".

\section{Step 5: Determine the optimal factor/level combination and confirm.}

Carry out the analysis of variance (ANOVA) on MPI values to identify the significant factors (process parameters). Compute level averages, i.e. average MPI values at different levels of the control factors. A larger MPI value implies better process performance and so selects the level averages of the influencing factors that lead to higher value for MPI. For other factors, any level may be chosen depending on the convenience. Finally, perform a confirmation

Experiment. 
Multi-response optimization of Wire-EDM process Using principal component analysis

\subsection{Results of WPCA}

\begin{tabular}{|l|l|l|l|l|l|}
\hline $\begin{array}{l}\text { Principal } \\
\text { components }\end{array}$ & Eigenvalue & $\begin{array}{l}\text { Proportion of } \\
\text { variation }\end{array}$ & cumulative & MRR & W \\
\hline First & 1.293 & 0.647 & 0.647 & 0.707 & 0.707 \\
\hline Second & 0.7070 & 0.353 & 1.000 & 0.707 & -0.707 \\
\hline
\end{tabular}

\begin{tabular}{|c|c|c|c|c|c|}
\hline \multirow[t]{2}{*}{ no } & \multicolumn{2}{|c|}{$\begin{array}{ll}\text { S/N } \\
\text { (nij) }\end{array}$} & \multicolumn{2}{|c|}{$\begin{array}{ll}\text { scaled } & \mathbf{S} / \mathbf{N} \\
\text { ratio (Y) } & \\
\end{array}$} & \multirow[t]{2}{*}{ MPI } \\
\hline & MRR & WWR & MMR & WWR & \\
\hline 1 & 33.4 & 23.2 & 0.22 & 0.45 & -0.11 \\
\hline 2 & 33.8 & 23.7 & 0.3 & 0.52 & 0.09 \\
\hline 3 & 35 & 21.2 & 0.49 & 0.18 & .188 \\
\hline 4 & 36.2 & 20.1 & 0.7 & 0.12 & 0.24 \\
\hline 5 & 34.8 & 23.9 & 0.5 & 0.49 & -0.09 \\
\hline 6 & 34.1 & 22.2 & 0.3 & 0.32 & -0.05 \\
\hline 7 & 36 & 23.1 & 0.59 & 0.45 & 0.02 \\
\hline 8 & 34.7 & 22.2 & 0.4 & 0.28 & 0.04 \\
\hline & 36.9 & 20 & 0.6 & 0.35 & 0.32 \\
\hline
\end{tabular}

"A larger value of MPI will imply better quality".

\section{Analysis of Variance for Means}

Factorial ANOVA is used

- General Linear Modal: MRR versus A, B, C, D

Analysis of Variance for MRR, using Adjusted SS for Tests

\begin{tabular}{|l|l|l|l|l|l|l|}
\hline source & DF & seq ss & Adj ss & Adj ms & F & P \\
\hline \hline A & 2 & 0.1156 & 0.115556 & 0.05778 & $* *$ & \\
\hline B & 2 & 0.0956 & 0.095556 & 0.04778 & $* *$ & \\
\hline C & 2 & 0.0022 & 0.002222 & 0.00111 & $* *$ & \\
\hline D & 2 & 0.0022 & 0.002222 & 0.00111 & $* *$ & \\
\hline ERROR & 0 & $*$ & $*$ & $*$ & & \\
\hline TOTAL & 8 & 0.2156 & & & & \\
\hline
\end{tabular}

\subsection{Analysis of Means}

The Analysis of Means (ANOM) was proposed by Dr. Ellis R. Ott (1967) as a logical extension of the Shewhart control charting technique for the purpose of graphically displaying the results from a designed experiment. Upper and Lower Decision Limits, along with a center line, provide the context for viewing the main effects and interactions.

In a standard control chart only the most recent data point is compared against the limits. The ANOM, on the other hand, is designed to provide an analysis with control on the overall Type I error rate. In essence, the ANOM combines the hypothesis testing features of ANOVA with the control chart's power of graphical presentation of the experiment means. ANOM is more powerful than ANOVA when only one mean is different from the others. Engineering significance is more easily assessed because both the magnitude and the direction of the effects are easily discernible from the plots. The underlying model for ANOM is the same for fixed effects ANOVA independent random samples from normal populations with homogeneous variances. 


\section{Optimum Parameters and their level}

\begin{tabular}{|l|l|l|}
\hline Rank & Parameters & Level \\
\hline 1 & T on & 7 \\
\hline 2 & T off & 8 \\
\hline 3 & Upper flush & 8 \\
\hline 4 & Servo feed & 7 \\
\hline
\end{tabular}

\section{Results \& discussion}

- $\mathrm{T}$ on is a most significant factor .

- UF is also a effective parameter.

- Toff \& WF less effective.

- Optimum experiment levels is -

\begin{tabular}{|l|l|l|l|l|}
\hline Sr.No & T-on & T-off & UF & Wire feed \\
\hline 1 & 7 & 8 & 8 & 7 \\
\hline
\end{tabular}

\section{Conclusion}

Wire electrical discharge machining (WEDM) processes require optimization of multiple performance characteristics (responses). The multiple responses of a WEDM process can be efficiently optimized using the weighted principal component (WPC) analysis method. Most importantly, there is no need for any input from the engineer(s) during analysis of the experimental data in the WPC method. The proposed WPC method reduces the uncertainty \& complexity of engineers judgment associated with taguchi method. Result shows that it can offer significantly better overall quality.

\section{References}

[1] Electronica machine tool ltd. Pune

[2] Non conventional machining processes by V.K.Jain

a.Journal Papers

[1] Ramkrishnan.R \& L.Karunamoorty, multioresponse optimization of WEDM operations using robust design of experiments, international journal of advan. Manufacturing tech. ,2006,29,105-112.

[2] Gauri S.K, Chakraborty S(2008)Multi Response Optimization for WEDM process Applying Weighted Principal component.23 ${ }^{\text {rd }}$ AIMTDR conf. 2008

[3] Liao \& Hung (2006) Multi Response Optimization for WEDM process Applying Weighted Principal component. international journal of advan. Manufacturing tech. 27,720-725.

[4] Sarkar S., Mitra S. Dutta S. and Bhatachryya B., 'Optimization of wire electrical discharge machining of $\gamma$-Titanium aluminide alloy', 21 st AIMTDR, VIT, Vellore (India), 20-22 Dec 2004, 397-402.

[5] Srinivasulu K, Varma D R S V, Sudhakara Reddy S, 'Experimental Investigation on the effect of machining parameters in wire-cut EDM of Inconel 718', 20 th AIMTDR, BIT, Mesra, Ranchi (India), 13-15 Dec 2002, 288-293.

[6] Tosun Nihat, Cogun Can, 'An Investigation on wire wear in WEDM', Journal of Material Processing Technology, 134 (2003) 273 278.

[7] Ranganath B J, Srikantappa A S, 'Effect of wire erosion rate on performance in Wire EDM', 20 th AIMTDR, BIT, Mesra, Ranchi (India), 13-15 Dec 2002, 281-287.

[8] Kalyanasiri, K., Boonmung S., "Effects of Wire-EDM machining variables on surface roughness of newly developed DC 53 die steel: Design of experiments and regression model" Journal of Material Processing Technology, 192-193 (2007) 459-464.

[9] Ramkrishnan R and Karunamoorthy, "Modelling and multi-response optimization of inconel 718 on machining of CNC WEDM process", Journal of Material Processing Technology, 207 (2008) 343-349.

[10] A Manna and B Bhattacharyya. 'Taguchi and Gauss Elimination Method : A Dual Response Approach for Parametric Optimization of CNC Wire Cut EDM of PRAISiCMMC'. International Journal of Advanced Manufacturing Technology, vol 28, 2006, p 67.

b.Books

[11] P J Ross. ‘Taguchi Technique for Quality Engineering'. McGraw-Hill, New York, 1989.

[12] Quality Engineering usin t design by Madhav g Robus Phadke.

c. links

[13] http://www.springerlink.com

[14] http://www.minitab.com

[15] http://www.electonicagroup.com

[16] http://www. Wikipedia.com

d. manual

[17] Operating manual of maxicut WEDM 